\title{
Myroides: A Rare but Hard-to-crack Villain in a Critical Care Setup
}

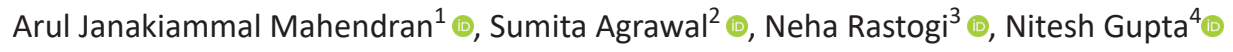

Keywords: Critically ill patients, Diabetes mellitus, Infectious diseases, Intensive critical care.

Indian Journal of Critical Care Medicine (2021): 10.5005/jp-journals-10071-23845

Editor Sir,

Myroides species are ubiquitous in the environment, yet infections are sporadic. They are of increasing concern since these bacteria are exceptionally resistant to most antibacterial agents. Given the corresponding increase in documented infections caused by Myroides species and the possible challenges with effective treatment, the clinicians should be wary of the possibility of this organism becoming a more prominent pathogen, especially to the immune-compromised population.

A 60-year-old male, known case of diabetes mellitus, had undergone left lower limb amputation for the diabetic foot. The next day, the patient presented to the intensive care unit with sudden onset breathlessness and fever. On examination, the patient was conscious and oriented. He was visibly tachypneic with a respiratory rate of 24 /minute; pulse was 130 /minute (tachycardia); and blood pressure (BP) was $90 / 54 \mathrm{~mm} \mathrm{Hg}$ with a mean arterial pressure (MAP) of $62 \mathrm{~mm} \mathrm{Hg}$. Arterial blood gas (ABG) analysis showed metabolic acidosis with a wide anion gap ( $\mathrm{pH}: 7.21, \mathrm{PO}_{2}: 110$, $\mathrm{PCO}_{2}: 16, \mathrm{HCO}_{3}: 12$, lactate: 4.5, $\mathrm{Na}+138$, and Chloride Levels (Cl)-94). Blood sugar was $354 \mathrm{mg} / \mathrm{dL}$, and urine for ketones was also positive. The patient started with treatment in lines of diabetic ketoacidosis (DKA) with intravenous fluids, potassium correction, bicarbonate, and antibiotics (meropenem, amikacin, and vancomycin) empirically in renal-modified doses.

Before initiation of antibiotics, urine and blood culture samples were withdrawn, ABG and BP improved over the next 24 hours, and sugar levels normalized. However, white blood cell count progressively increased from baseline 18,000 to $24,000 / \mathrm{mm}^{3}$, and the patient was having spikes of fever. It was concluded that DKA had resolved, but sepsis persisted. At this point, urine routine showed 45 to 50 pus cells, chest X-ray was normal, wound site was healthy, and urine culture awaited. The patient's Foley's catheter was changed and antibiotics upgraded to colistin since most of the local flora causing hospital-acquired infection were sensitive only to colistin.

Nevertheless, the patient continued to have a high-grade fever over the next 48 hours. On day 3, the blood culture report was available, which was negative. A urine culture on day 2 showed gram-negative rods; the final report of the urine culture revealed Myroides species, VITEK 2 automated diagnostic system. However, sub-speciation with $16 \mathrm{~S}$ rRNA sequencing was not available in the author's center. The isolate showed resistance to all broad-spectrum antibiotics, including colistin - a repeat sensitivity analysis ordered for sensitivity to cotrimoxazole, tetracyclines, tigecycline, and minocycline. The report showed sensitivity to only minocycline.

\author{
${ }^{1,4}$ Department of Pulmonary, Critical Care and Sleep Medicine, VMMC \\ and Safdarjung Hospital, Delhi, India \\ ${ }^{2}$ Department of Pulmonary, Critical Care and Sleep Medicine, \\ Medipulse Hospital, Jodhpur, Rajasthan, India \\ ${ }^{3}$ Department of Infectious Diseases, AlIMS, Delhi, India
}

Corresponding Author: Sumita Agrawal, Department of Pulmonary, Critical Care and Sleep Medicine, Medipulse Hospital, Jodhpur, Rajasthan, India, e-mail: doc.sumitaagrawal@gmail.com

How to cite this article: Mahendran AJ, Agrawal S, Rastogi N, et al. Myroides: A Rare but Hard-to-crack Villain in a Critical Care Setup. Indian J Crit Care Med 2021;25(6):735-736.

Source of support: Nil

Conflict of interest: None

The patient received minocycline $200 \mathrm{mg}$ intravenous loading dose followed by $100 \mathrm{mg}$ intravenous twice daily for three days, after which his fever subsided, and the TLC count decreased. Repeat urine routine reports returned normal, followed by sterile culture reports. Subsequently, the patient was discharged in stable condition.

Myroides species are a rare cause of human infection. Infections are rare but can occasionally be life-threatening. ${ }^{1}$ The clinical manifestation of Myroides causing urinary tract infection was documented in patients with chronic nephritis, urinary retention, urinary calculi, and diabetes mellitus. ${ }^{2,3}$ Reports of isolated outbreaks of urinary tract infections following exposure to a contaminated water source or in the trauma setting are reported in the literature. ${ }^{1,4}$ The two most common Myroides species seen in humans are $M$. odoratus and $M$. odoratimimus. Other less common Myroides species, such as M. pelagicus, M. profundi, and M. marinus, have not been reported as a cause of infections in humans. ${ }^{3-5}$ The traditional epidemiology of Myroides involves infection of an immune-compromised host; diabetes in index case represents the same. Both $M$. odoratus and $M$. odoratimimus infections in people with diabetes were previously documented..$^{6-8}$ In the index case, the primary portal of entry and infection source postulated to be the presence of Foley's catheter, possibly because of the strong tendency of Myroides spp. to form biofilms. In the case of Myroides urinary tract infection, the susceptibility to various antibiotics reported in the literature is quite variable. Therefore, choosing the appropriate antimicrobial treatment for Myroides infections can be quite challenging because of the limited clinical experience. However, most reports describe the Myroides isolates as multi- or pan-drug-resistant and challenging to treat and, therefore, with variable success. Solanki et al. ${ }^{8}$ reported that all the isolates of Myroides recovered from urine were resistant to all the 
antibiotics tested. Verma et al. ${ }^{9}$ reported successful treatment of Myroides infection sensitive only to minocycline, as seen in the current discussion. Myroides remain an unusual pathogen that is omnipresent in the environment. Nevertheless, frequently described in a variety of clinical settings. Clinicians should remain wary of the plausibility of this pathogen as an etiologic agent for invasive infection, notably in the immune-compromised or in the inadequacy of response from routine treatment.

\section{ORCID}

AJ Mahendran ㄴ) https://orcid.org/0000-0003-4907-1312

Sumita Agrawal 니 https://orcid.org/0000-0001-5713-6556

Neha Rastogi (1) https://orcid.org/0000-0002-0063-3444

Nitesh Gupta 으 https://orcid.org/0000-0002-5842-5584

\section{References}

1. Benedetti P, Rassu M, Pavan G, Sefton A, Pellizzer G. Septic shock, pneumonia, and soft tissue infection due to Myroides odoratimimus: report of a case and review of Myroides infections. Infection 2011;39(2):161-165. DOI: 10.1007/s15010-010-0077-1.

2. Holmes B, Snell JJ, Lapage SP. Flavobacterium odoratum: a species resistant to a wide range of antimicrobial agents.J Clin Pathol 1979;32(1):73-77. DOI: 10.1136/jcp.32.1.73.
3. Hu SH, Yuan SX, Qu H, Jiang T, Zhou YJ, Wang MX, et al. Antibiotic resistance mechanisms of Myroides sp. J Zhejiang Univ Sci B 2016;17(3):188-199. DOI: 10.1631/jzus.B1500068.

4. Yağci A, Cerikçioğlu N, Kaufmann ME, Malnick H, Söyletir G, Babacan $\mathrm{F}$, et al. Molecular typing of Myroides odoratimimus (Flavobacterium odoratum) urinary tract infections in a Turkish hospital. Eur J Clin Microbiol Infect Dis 2000;19(9):731-732. DOI: 10.1007/s100960070001.

5. Cho SH, Chae SH, Im WT, Kim SB. Myroides marinus sp. nov., a member of the family Flavobacteriaceae, isolated from seawater. Int J Syst Evol Microbiol 2011;61(Pt 4):938-941. DOI: 10.1099/ijs.0.024067-0.

6. Motwani B, Krezolek D, Symeonides S, Khayr W. Myroides odoratum cellulitis and bacteremia: a case report. Infect Dis Clin Pract 2004;12(6):343-344. DOI:10.1097/01.idc.0000144904.51074.79.

7. Endicott-Yazdani TR, Dhiman N, Benavides R, Spak CW. Myroides odoratimimus bacteremia in a diabetic patient. Proc (Bayl Univ Med Cent) 2015;28(3):342-343. DOI: 10.1080/08998280.2015.11929268.

8. Solanki R, Dawar R, Aggarwal DK, Rani H, Imdadi F, Jasuja S. Nonfermenting gram-negative bacilli and urinary tract infectionsorting the mystery of infections caused. J Med Microb Diagn 2015;4(4):21. DOI: 10.4172/2161-0703.1000210.

9. Verma Y, Hemachander SS, Shah K. Urinary tract infection caused by Myroides spp. In diabetic patients: to be or not to be. Indian J Case Rep 2018;4(1):1-3. DOI: 10.32677/IJCR.2018.v04.i01.001 